\title{
Company Profile
}

\section{Olympus \\ Soft Imaging Solutions}

As a "Center of Competence and Excellence," Olympus Soft Imaging Solutions (OSIS) is responsible for the development, production, distribution, support, and service of micro-imaging software and hardware system within the Olympus Group. And OSIS is one of the most successful global providers of transmission and scanning electron microscopy equipment. Since 1986 our products have attained a well-respected position on the worldwide electron microscopy market in all areas and applications.

\section{TEM and SEM software}

iTEM and Scandium are our image analysis platforms for transmission electron microscopy and scanning electron microscopy, respectively. With their extensive range of tools and options, efficiency is delivered whatever your tasks and applications are. Solution-oriented extension packages offer an efficient means of expanding the capabilities of the base versions.

\section{TEM cameras}

Our TEM camera line-up of side- and bottom-mounted cameras reflects the current requirements of life sciences and materials analysis. Properties such as large field of view, outstanding sensitivity, fast frame rates, excellent signal-to-noise ratio, easy installation, automatic on-screen focusing, and automatic image enhancement all guarantee that the TEM workday is efficient. Aside from their quality and affordability, all cameras are completely integrated in iTEM.

Olympus Soft Imaging Solutions is active around the world. Furthermore a team of authorized sales partners is responsible for product sales, including consultation, installation, training courses, and support.

\section{OLYMPUS}

Your Vision, Our Future
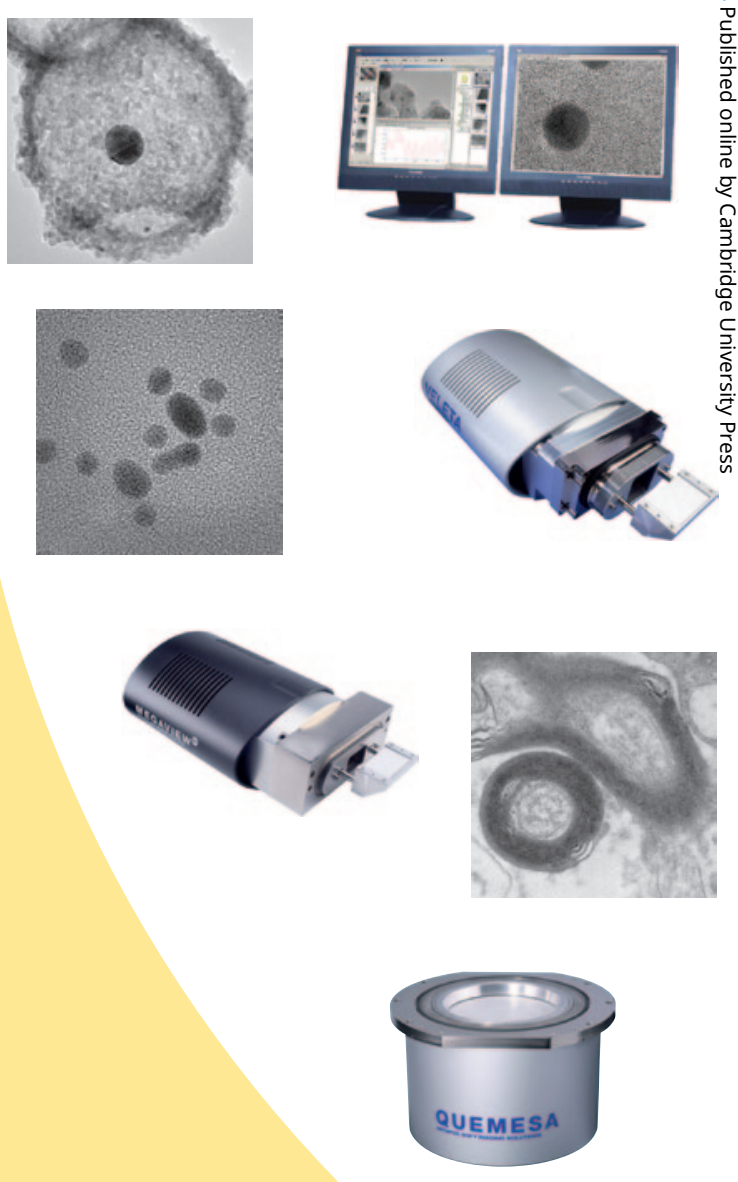

\section{How to find us}

Olympus Soft Imaging Solutions is active around the world and currently operates offices in Europe and the United States.

Olympus Soft Imaging Solutions

Johann-Krane-Weg 39

48149 Münster

Germany

Tel: +49251 798000

Fax: +49251798006060

Info.osis@olympus-sis.com

www.soft-imaging.net

For information about your local representative or any other information, please see our website and don't hesitate to contact us. 